\title{
ROAD RECOGNITION BASED ON DECISION LEVEL FUSION OF SAR AND OPTIC DATA
}

\author{
M. Lazari Zare, F. Tabib Mahmoudi* \\ Dept. of Geomatic Engineering, Civil Engineering Faculty, Shahid Rajaee Teacher Training University, Tehran, Iran - \\ mohadeseh.zare21@gmail.com, fmahmoudi@sru.ac.ir
}

Commission VI, WG VI/4

KEY WORDS: Road recognition, Decision level fusion, Support vector machine, SPOT image, SAR

\begin{abstract}
:
Road recognition and extraction based on remotely sensed data is efficient and applicable in much urban management studies. In this research, the capabilities of SPOT and SAR images are investigated for road recognition. Spectral and textural similarities between roads and other urban objects such as building's roofs many cause some difficulties in road recognition based on SPOT image. On the other hand, SAR images are good for small road recognition but, may have some difficulties for detecting roads among vegetation. The proposed method in this paper is a decision level fusion of SPOT and SAR classification results in order to modify extracted road regions. This method has three main steps; 1) texture feature extraction from each of the SPOT and SAR images, 2) classifying each of the SPOT and SAR images based on SVM classifier, 3) decision level fusion of classification results in order to reduce road recognition difficulties and having optimum road regions. Performing the capabilities of the proposed decision level fusion algorithm for road recognition can improve the quality of the classification for about $21 \%$.
\end{abstract}

\section{INTRODUCTION}

Road network in urban areas may change due to natural disasters (such as floods and earthquakes) or urban sprawl. Updating roads in urban city models is useful for many applications, such as emergency assistance, spatial analysis and routing. Road recognition based on remotely sensed data is an efficient strategy.

High resolution optic and SAR images have been successfully used for road recognition (Lisini et al., 2006; Mohammadzadeh et al.,2006; Negri et al.,2006; Wang et al.,2016; Wang et al., 2005). Optic images are more successful than SAR in detecting background pixels. But, three are some difficulties due to spectral and textural similarities between roads and other urban objects such as building's roofs.

Mohammadzadeh et al., 2006 proposed a method based on fuzzy logic and mathematical morphology in order to extract main road centrelines from pan-sharpened IKONOS images (Mohammadzadeh et al., 2006).

Wang et al., 2016 proposed a knowledge-based method for road recognition based on optic data. This method incorporates the spatial texture features into urban road extraction. The spatial texture feature is initially extracted by the local Moran's I. Features are selected to form the hypothesis and verification model based on road knowledge. Finally, roads are extracted by applying the hypothesis and verification model and are postprocessed based on the mathematical morphology (Wang et al., 2016).

Wang et al., 2005 proposed a road extraction method based on a pan-sharpened QuickBird image. The fuzzy k-means clustering is applied on pan-sharpened image in order to identify the road class that may include non-road objects. Applying the Canny operator to the panchromatic image in order to detect the edges, as well as using morphological operators and baseline-based thresholds, along with the directional texture detection were used to modify the extracted road regions (Wang et al.,2005).

On the other hand, in SAR images high buildings near to roads have the role of corner reflectors. Therefore, one can better recognize buildings and roads in SAR images. Another advantage of SAR images is the capability of imaging all day long, which is not dependent on weather conditions (Khesali et al.,2014).

Negri et al., 2006 proposed an urban road network extraction method based on high-resolution synthetic aperture radar images. It is based on novel street candidate detection method, which was further specified into a multi scale feature fusion detector and a segment extractor, followed by optimization using a Markov Random Field description of the road network (Negri et al., 2006).

Lisini et al.,2006 proposed a road extraction method based on the fusion of classification (statistical information) and line detection (structural information). Then, a modified Markovian approach was used for solving the global network optimization problem (Lisini et al.,2006).

Fusion of optic images and SAR data can complete knowledge space for road recognition applications. Some researches were used data fusion algorithms for road recognition based on various remotely sensed images such as SAR and optic data (Lisini et al.,2011; Khesali et al,2014).

Lisini et al.,2011 proposed road extraction in wide area images, obtained by satellite sensors in both the optical/infrared and microwave domains. This study is aimed at bridging these gaps and provides a unique framework for the extraction of roads with different characteristics using optical or SAR data sets. The approach exploits a multi-scale analysis to adapt to the different resolutions of data and a pre-processing step to adapt to the different wavelengths of data. When possible, the framework allows the fusion of the road networks extracted from optical

\footnotetext{
* Corresponding author
} 
and SAR data of the same area. The soundness of the approach is proved by means of the analysis of Landsat and ALOS data of an area in Congo (Lisini et al.,2011).

Khesali et al, 2014 proposed a data fusion algorithm for road extraction from high spatial resolution radar and optics images. TerraSAR-X and IKONOS images were used for this purpose. After performing image registration, texture features were extracted from both the images and classified using neural network. After comparing the output images obtained from each image, it shows that road recognition has some difficulties in both of the SAR and optic images. Given the complementary capabilities of the two images in path extraction, the fusion of these two sources is an efficient way of developing algorithms (Khesali et al, 2014).

In this study, a decision level fusion algorithm is proposed for road recognition based on classifier fusion of SPOT and SAR images.

\section{PROPOSED ROAD RECOGNITION METHOD}

The proposed road recognition method in this paper is a decision level fusion algorithm based on the pixel based support vector machine (SVM) classification results of SPOT and SAR images. As it is illustrated in figure 1, this method has three main steps;

1) Texture feature extraction from each of the SPOT and SAR images,

2) Classification of SPOT and SAR images based on SVM classifier,

3) Classifier fusion or decision level fusion of SPOT classification result and SAR classification result in order to have optimum roads.

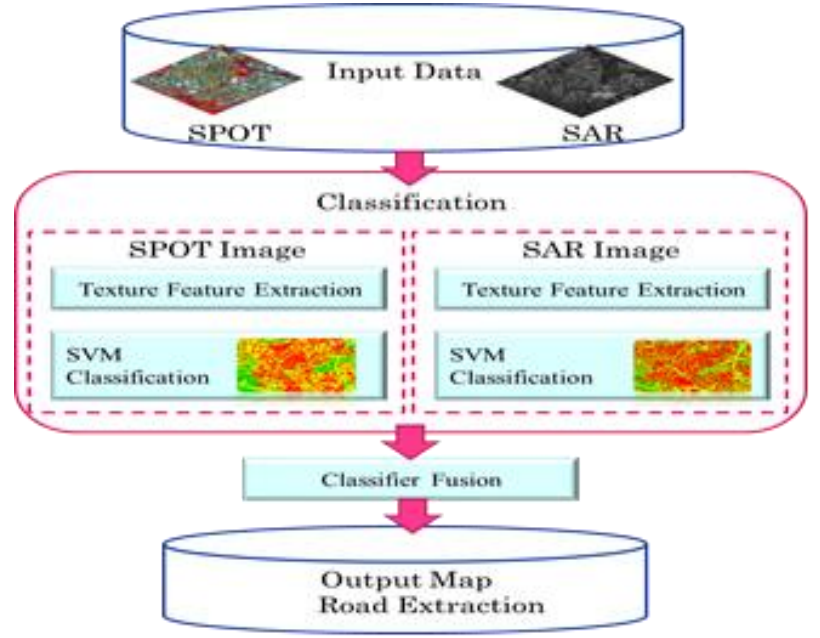

Figure 1. Structure of the proposed road recognition method

In the following sections of this paper, texture feature extraction and SVM classification are explained for each of the SPOT and SAR images, separately.

\subsection{Texture Feature Extraction}

Textural features are measured based on grey values relationships in a local neighbouring window of the remotely sensed images. Energy, entropy, homogeneity, contrast, mean, dissimilarity and etc. are some of the most popular texture features in object recognition researches. Texture feature extraction relates to the type of object and the characteristics of the image.
In this paper, mean, homogeneity, dissimilarity and contrast those are measured in grey level co-occurrence matrix (GLCM) space are used as feature space for each band of SPOT image. The above mentioned texture features are selected based on diversity analysis and visual inspection of an expert operator for recognition of roads among other urban objects. Table 1 depicts mathematical basis of some the above mentioned textural features.

\begin{tabular}{|l|l|}
\hline Feature Name & Formula \\
\hline Mean & $\sum_{i, j=0}^{N-1} i \times P(i, j)$ \\
\hline Homogeneity & $\sum_{i, j=0}^{N-1} \frac{P_{i, j}}{1+(i+j)^{2}}$ \\
\hline Contrast & $\sqrt{\frac{1}{M N} \sum_{i=0}^{N-1} \sum_{j=0}^{M-1}\left(I_{i j}-\bar{I}\right)^{2}}$ \\
\hline
\end{tabular}

Table 1. Mathematics of the measured textural features on SPOT image

Different characteristics of SAR images lead to selection of the different textural features for road recognition. Mean and data range are selected as optimum textural features for classification of SAR image by an expert analysis.

\subsection{Support Vector Machine Classification}

Support Vector Machine (SVM) classifier with RBF kernel is applied on both of the SPOT and SAR images, separately.

For SPOT, layer stacking of the mean, homogeneity, dissimilarity and contrast features those are measured for each band of image together with three spectral image bands, generates 15 layers as input feature space for SVM classifier. For SAR image, the optimum selected features mean and data ranges are stacked and generates 3 layers input feature space for SVM classification of SAR.

SVM parameters were illustrated in tables 2 and 3 for SPOT and SAR classification, respectively.

\begin{tabular}{|l|c|}
\hline \multicolumn{1}{|c|}{ Parameter's Name } & Value \\
\hline Gamma & 0.8 \\
\hline Penalty parameter & 100 \\
\hline Classification Probability Threshold & 0.2 \\
\hline
\end{tabular}

Table 2. SVM parameters for SPOT image classification

\begin{tabular}{|l|c|}
\hline \multicolumn{1}{|c|}{ Parameter's Name } & Value \\
\hline Gamma & 4 \\
\hline Penalty parameter & 200 \\
\hline Classification Probability Threshold & 0.01 \\
\hline
\end{tabular}

Table 3. SVM parameters for SAR image classification

\subsection{Decision Level Fusion}

Classification result of SPOT image has some errors due to spectral and textural similarities between roads and building's roofs. On the other hand, classification of SAR image may have some difficulties in recognition of roads and vegetation.

Classification and recognition of small and narrow roads in dense urban areas have weak results in SPOT but, in SAR results are more reasonable. Therefore, performing decision level fusion between the classification results of SPOT and SAR 
images can improve overall accuracy of the generated classification map.

Knowledge based rules based on structural features and contextual relationships between roads and other objects in urban areas are used as a classifier fusion strategy in this paper. In fact, a decision level fusion is performed based on size, shape and neighbourhood relationships of road regions in the classification results of SPOT and SAR images. Some of the pre-defined rules are explained:

1) For pixel $(i, j)$, IF SAR classification $(i, j)=$ Road and SPOT classification $(i, j)=$ Building and Elongation of the region included pixel $(i, j)$ in SPOT classification result >threshold THEN $(\mathrm{i}, \mathrm{j})$ belongs to Road object class.

2) For pixel $(i, j)$, IF SPOT classification $(i, j)=$ Vegetation and SAR classification $(i, j)=$ Building OR ROAD and Roundness of the region included pixel $(i, j)$ in SAR classification result $>$ threshold THEN $(\mathrm{i}, \mathrm{j})$ belongs to Vegetation object class.

3) For pixel $(i, j)$, IF SAR classification $(i, j)=\operatorname{Road}$ and SPOT classification $(i, j)=$ Building and Rectangular fit of the region included pixel $(i, j)$ in SPOT classification result <threshold THEN (i,j) belongs to Road object class.

\section{EXPERIMENTAL RESULTS}

\subsection{Study Area and Dataset}

The potential of the proposed road recognition methodology is evaluated for SPOT and SAR data over an urban area in Barcelona, Spain. The utilized SAR data were collected on May 2011 with $2.5 \mathrm{~m}$ spatial resolution. The SAR data is Terra SAR$\mathrm{X}$ Strip map with $\mathrm{HH}$ polarization channel, and its angle of incidence is equal to 35.2. SPOT 5 image is also pan-sharpened with $2.5 \mathrm{~m}$ spatial resolution (Figure 2).
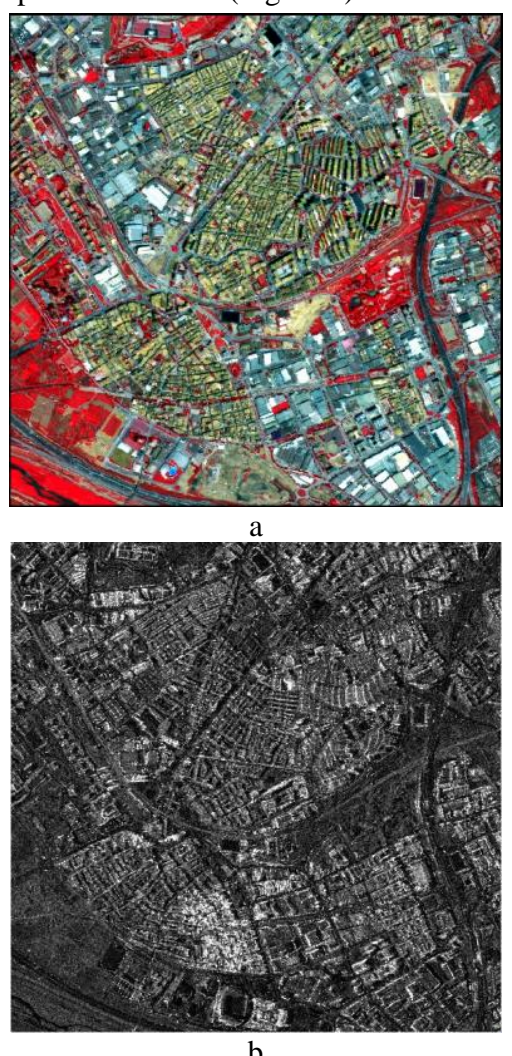

Figure 2 image, a) SPOT, b) SAR image
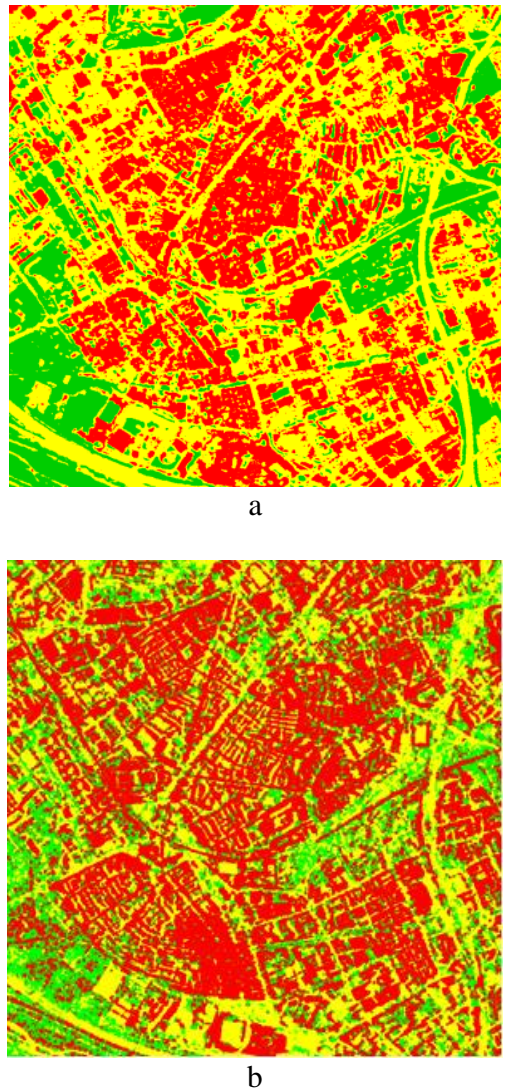

Figure 3. SVM classification result on a) SPOT, b) SAR

\subsection{Obtained Results}

According to the nature of the urban areas, building, road and vegetation are defined as three main object classes.

For SVM classification of SPOT image, 5096 pixels of roads, 5949 pixels of buildings and 4653 pixels of vegetation objects are selected as training data of the algorithm.

Before texture feature extraction from SAR, Erod and Gama filters are applied on SAR image in order to reduce speckle noise. Then, 2203 pixels of roads, 5144 pixels of buildings and 1358 pixels of vegetation objects are selected from SAR image as training samples for SVM classification.

The obtained results from performing SVM classifier on SPOT and SAR images are illustrated in figure 3. Spectral and textural similarities between roads and building's roofs led to some difficulties in road and building object recognition classes in SPOT classification map. Vegetation and long roads have better recognition results for SPOT.

On the other hand, the various nature of SAR image leads to some difficulties in vegetation recognition. However, narrow and small roads near buildings in complex urban areas have better recognition results for SAR.

Therefore, performing decision level fusion between SAR and SPOT classification results may produce more accurate recognition map. Using pre-defined thresholds for size, elongation and rectangularity of the road regions, knowledge based decision fusion strategy can improve road recognition results. In figure 4 the improvements of the obtained results from decision fusion are illustrated in some selected samples of the study area. 

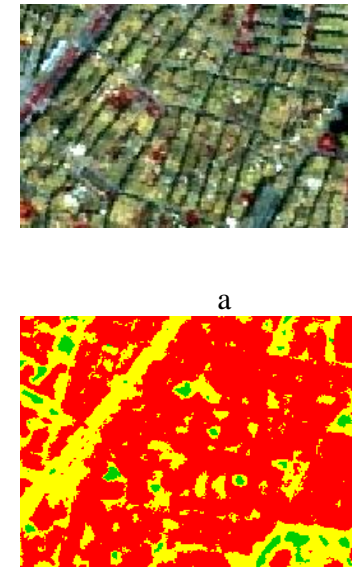

c
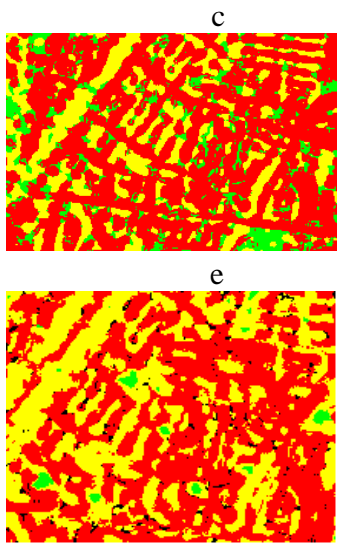

$\mathrm{g}$
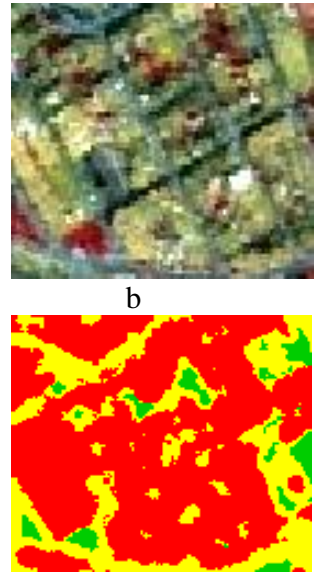

d
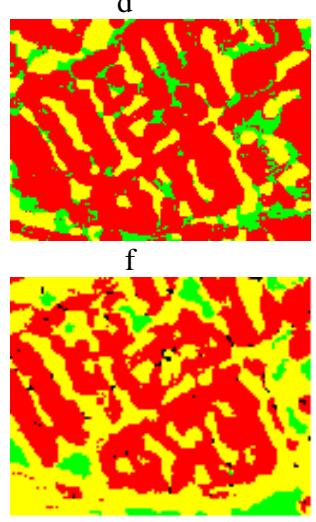

$\mathrm{h}$

Figure 4. selected sample areas; a,b) SPOT image, c,d) SPOT classification result, e,f) SAR classification result, g,h) decision fusion result

\subsection{Accuracy Assessment}

For the accuracy assessment of the obtained results from the proposed road recognition algorithm, generated classification maps of SPOT and SAR images are evaluated separately based on pre-defined ground truth. Figure 5 depicts the comparison between the overall accuracies of the generated SPOT and SAR classification maps and the result of classifier fusion of them.

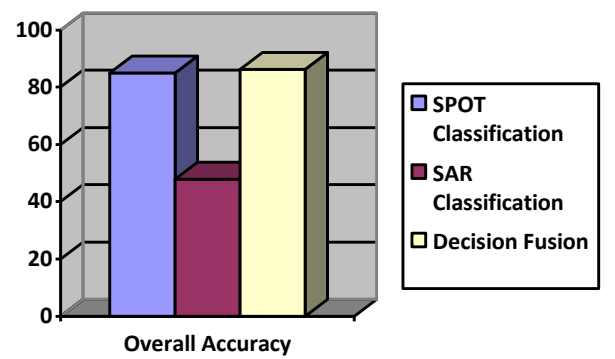

Figure 5. Comparing overall accuracies of the classification maps and decision fusion of them

Performing decision fusion between the SVM classification results of SPOT and SAR images can improve the overall accuracy of the final classification map for about $2 \%$. Moreover, the classification quality of the SPOT image is

compared with the quality of the final decision fusion classification map (See equation1 for classification quality).

$$
\text { Quality }=\frac{\text { TruePositive }}{\text { TruePositive }+ \text { FalsePositive }+ \text { FalseNegative }}
$$

Where True Positive denotes the extracted road pixels those coincide with the reference data, False Negative refers to the road pixels those are in the reference data but not in the extracted results and False Positive represents the mistakes in road extraction results. As it is depicted in table 4, the classification quality of the road objects are improved for about $21 \%$ after performing decision fusion of SAR and SPOT classification maps.

\begin{tabular}{|c|c|c|c|}
\hline & \multicolumn{3}{|c|}{ Object classes } \\
\cline { 2 - 4 } & Road & Building & Vegetation \\
\hline SPOT data & $\mathbf{5 5 . 7 6}$ & 73.77 & 99.28 \\
\hline Fusion result & $\mathbf{7 6 . 6 9}$ & 68.09 & 99.98 \\
\hline
\end{tabular}

Table 4. Quality of the SPOT classification and decision fusion results

\section{CONCLUSION}

Updating roads in urban city models is useful for many applications, such as emergency assistance, spatial analysis and routing. Therefore, performing road recognition based on remote sensing data is one of the research topics. In this paper, a decision level fusion algorithm is proposed for road recognition based on SPOT and SAR images. The proposed decision fusion method is a classifier fusion based on SVM pixel classification results of input images. Performing decision level fusion on the SVM classification maps of SPOT and SAR images could improve the classification quality of roads for about $21 \%$.

Moreover, using the various characteristics of SAR image improved the recognition results of small and narrow road regions in the final classification map.

\section{REFERENCES}

Khesali, E. Valadan Zoej, M. Dehghani, M. Mokhtarzade, M.,2014,Comparison of road extraction in urban areas from high resolution TerraSAR-X and IKONOS images using texture Features in neural network Algorithms, Iranian Remote Sensing \& GIS ,Vol.5, No.4.

Lisini, G. Tison, C. Tupin, F and Gamba, P.,2006, Feature fusion to improve road network extraction in high-resolution SAR images, IEEE Geoscience and Remote Sensing Letters, vol. 3 , no. 2 .

Lisini, G. Gamba, P. Dell'Acqua, F and Holecz, F.,2011, First results on road network extraction and fusion on optical and SAR Images using a multi-scale adaptive approach, Taylor \& Francis,Vol. 2, No. 4,PP. 363-375.

Mohammadzadeh, A. Tavakoli, A. and ValadanZoej, M.J., 2006, Road Extraction based on Fuzzy Logic and Mathematical Morphology from Pan-Sharpened IKONOS Images, The Photogrammetric record 21(113), PP. 44-60. 
Negri, M. Gamba, P. Lisini, G and Tupin, F.,2006,

Junction-aware Extraction and regularization of urban road networks in highresolution SAR images, IEEE Transactions on Geoscience and Remote Sensing, vol. 44, no. 10.

Wang, J. Qin, Q. Gao, ZH. Zhao, J and Ye, X., 2016,A New Approach to Urban Road Extraction Using high-Resolution Aerial Image, ISPRS Int. J. Geo-Inf. 2016, 5, 114; doi:10.3390/ijgi5070114.

Wang, R. Hu, Y and Zhang, X., 2005, Extraction of road networks using pan-sharpened multispectral and panchromatic quickbird images, Geomatica. Vol. 59, No. 3, PP. 263-274. 